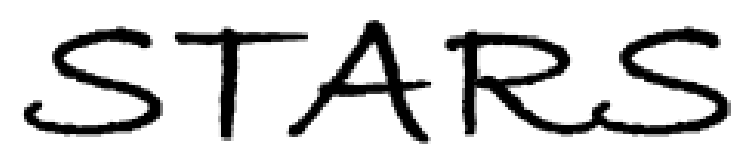

University of Central Florida

STARS

Faculty Bibliography 2000s

Faculty Bibliography

$1-1-2006$

\title{
Nuclear spectra of comet 162P/Siding Spring (2004 TU12)
}

Humberto Campins

University of Central Florida

Julie Ziffer

University of Central Florida

Javier Licandro

Noemí Pinilla-Alonso

Yanga Fernández

University of Central Florida

See next page for additional authors

Find similar works at: https://stars.library.ucf.edu/facultybib2000

University of Central Florida Libraries http://library.ucf.edu

This Article is brought to you for free and open access by the Faculty Bibliography at STARS. It has been accepted for inclusion in Faculty Bibliography 2000s by an authorized administrator of STARS. For more information, please contactSTARS@ucf.edu.

\section{Recommended Citation}

Campins, Humberto; Ziffer, Julie; Licandro, Javier; Pinilla-Alonso, Noemí; Fernández, Yanga; León, Julia De; Mothé-Diniz, Thais; and Binzel, Richard P., "Nuclear spectra of comet 162P/Siding Spring (2004 TU12)" (2006). Faculty Bibliography 2000s. 4658.

https://stars.library.ucf.edu/facultybib2000/4658

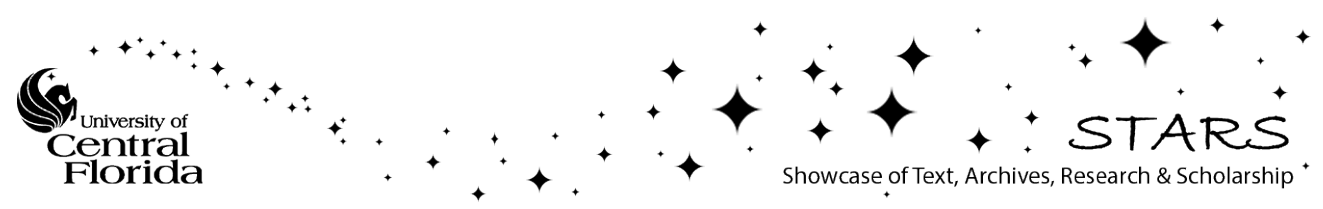


Authors

Humberto Campins, Julie Ziffer, Javier Licandro, Noemí Pinilla-Alonso, Yanga Fernández, Julia De León, Thais Mothé-Diniz, and Richard P. Binzel 


\title{
NUCLEAR SPECTRA OF COMET 162P/SIDING SPRING (2004 TU12)
}

\author{
Humberto Campins, ${ }^{1,2,3}$ Julie Ziffer, ${ }^{1}$ Javier Licandro, ${ }^{3,4,5}$ Noemí Pinilla-Alonso, ${ }^{6}$ Yanga Fernández, ${ }^{1,3,7}$ \\ Julia de León, ${ }^{5}$ Thais Mothé-Diniz, ${ }^{8}$ and Richard P. Binzel ${ }^{3,9}$ \\ Received 2006 March 8; accepted 2006 May 25
}

\begin{abstract}
We present visible and near-IR spectra of the nucleus of comet 162P/Siding Spring (also known as 2004 TU12) obtained in 2004 December, while it had no detectable coma. This is the third object observed to have intermittent cometary activity even when relatively close to the Sun. The spectra show no strong features in this wavelength range. This paucity of deep absorptions is common among low-albedo asteroids and the few comet nuclei observed in this spectral region. Marginal spectral structure is observed in the visible spectrum, and beyond $2 \mu \mathrm{m}$ the flux from the nucleus is dominated by thermal emission. We compare the spectrum of $162 \mathrm{P}$ with published spectra of other comet nuclei, primitive asteroids, and meteorites. Comet nuclei display a range of spectral shapes and slopes not unlike those observed among outer main-belt asteroids but closest to Trojan asteroids. No suitable spectral matches to comet 162P were found among primitive (chondritic) meteorites. We modeled our visible and near-IR spectra using the scattering theory described by Shkuratov et al. (1999), and our approach is similar to that used by Emery and Brown for modeling Trojan asteroids. Our best fits to the spectral shape and albedo include mixtures containing amorphous carbons, organics, and silicates. The absence of strong spectral features prevents the identification of specific minerals, and the resulting model compositions are not unique. The observations beyond $2 \mu \mathrm{m}$ are interpreted in a companion publication by Fernández and coworkers.
\end{abstract}

Key words: comets: individual (162P) — infrared: solar system

Online material: color figure

\section{INTRODUCTION}

Comet-asteroid transition objects provide opportunities to study physical and dynamical processes that are less evident or absent in the more clear-cut members of either population. Until recently, the two types of transitional objects were inactive or low-activity comets or asteroids in comet-like orbits (e.g., Weissman et al. 2002). Comets with intermittent activity join the other two types of transitional objects as important pieces of this puzzle. Comet $162 \mathrm{P} /$ Siding Spring, also known as 2004 TU12, is the third inner solar system object observed to have intermittent cometary activity even when relatively close to the Sun. The other two such objects are comets 107P/Wilson Harrington and 133P/Elst-Pizarro, also known as asteroids 4015 and 7968, respectively (Weissman et al. 2002; Hsieh et al. 2004 and references therein). This object was discovered on UT 2004 October 10 at Siding Spring, Australia, by R. McNaught (MPEC 2004-T55). At that time, one month before its perihelion, the appearance was asteroidal. It was first recognized as a comet on 2004 November 12, when an eastward tail was reported (Mallia et al. 2004); however, the nuclear image appeared stellar. The length and brightness of the tail has

\footnotetext{
${ }^{1}$ Department of Physics, University of Central Florida, P.O. Box 162385, Orlando, FL 32816-2385; campins@physics.ucf.edu.

${ }^{2}$ Lunar and Planetary Laboratory, University of Arizona, Tucson, AZ 85721.

3 Visiting Astronomer at the Infrared Telescope Facility, which is operated by the University of Hawaii under contract to the National Aeronautics and Space Administration.

${ }^{4}$ Isaac Newton Group of Telescopes, P.O. Box 321, 38700 Santa Cruz de La Palma, La Palma, Spain.

5 Instituto de Astrofísica de Canarias, c/Vía Láctea s/n, 38200 La Laguna, Tenerife, Spain.

${ }^{6}$ Fundación Galileo Galilei and Telescopio Nazionale Galileo, P.O. Box 565, 38700 Santa Cruz de La Palma, Tenerife, Spain.

7 Institute for Astronomy, University of Hawaii, Honolulu, HI 96822.

8 Observatorio Nacional, Rio de Janeiro, Brazil.

9 Department of Earth, Atmospheric, and Planetary Sciences, Massachusetts Institute of Technology, Cambridge, MA 02139.
}

been variable, but the nucleus has remained stellar. The tail was originally reported on November 12.0 and 12.8 to be about $2^{\prime}$ and $4^{\prime}$ long, respectively, and at a position angle of $70^{\circ}$. The tail was observed to fade and detach from the nucleus over the following few days (Masi 2004). The tail reappears in images taken by C. Hergenrother on UT 2004 December 9 and by J. Lacruz on UT 2004 December 10 (C. Hergenrother 2005, private communication). On November 12 and December 9 the heliocentric $(R)$ and geocentric $(\Delta)$ distances were $R=1.23$ and $1.27 \mathrm{AU}$ and $\Delta=0.46$ and $0.61 \mathrm{AU}$, respectively. The orbital elements of $162 \mathrm{P}$ make it a Jupiter-family comet with a Tisserand invariant $T=2.80$. The Tisserand invariant is a constant of the motion in the restricted three-body problem (with Jupiter). This parameter is often used to differentiate dynamically between Jupiter-family comets $(2<T<3)$, most asteroids $(T>3)$, and Halley-type comets $(T<2)$ (e.g., Carusi et al. 1987; Levison 1996).

In this work we present near-IR and visible spectroscopy of comet 162P obtained in 2004 December, approximately 2 months after its discovery. We use these observations to characterize the nuclear surface of this comet and to compare it with other similarly observed comet nuclei, primitive asteroids, and meteorites. Mid-IR $(8-25 \mu \mathrm{m})$ photometry is presented in Fernández et al. (2006, hereafter Paper II) and used to estimate the size, albedo, and other thermal properties of the nucleus of 162P.

\section{OBSERVATIONS}

We obtained near-IR $(0.8-2.4 \mu \mathrm{m})$ and visible spectra of 162P. The observing geometry for these dates and the instruments used are presented in Table 1.

\subsection{Near-Infrared Spectra}

We obtained three low-resolution near-IR spectra of 162P from two telescopes. On UT 2004 December 3.25 and 10.25 we used the NASA $3.0 \mathrm{~m}$ Infrared Telescope Facility (IRTF) with the SpeX instrument. On UT 2004 December 11.88 we observed 
TABLE 1

Observing Dates and Orbital Geometry for 162P

\begin{tabular}{|c|c|c|c|c|c|}
\hline UT Date 2004 & $\begin{array}{c}R \\
(\mathrm{AU})\end{array}$ & $\begin{array}{c}\Delta \\
(\mathrm{AU})\end{array}$ & $\begin{array}{c}\text { Solar Phase Angle } \\
\text { (deg) }\end{array}$ & Air Mass & Instrument and Telescope \\
\hline Dec $3.25 \ldots \ldots \ldots \ldots \ldots \ldots$ & 1.26 & 0.57 & 49 & 1.05 & SpeX IRTF \\
\hline Dec $10.25 \ldots \ldots \ldots \ldots \ldots$ & 1.28 & 0.63 & 49 & 1.05 & SpeX IRTF \\
\hline Dec $11.88 \ldots \ldots \ldots \ldots \ldots$ & 1.28 & 0.64 & 48 & 1.10 & NICS TNG \\
\hline Dec $12.86 \ldots \ldots \ldots \ldots \ldots \ldots \ldots$ & 1.29 & 0.65 & 48 & 1.10 & ALFOSC NOT \\
\hline
\end{tabular}

from the $3.56 \mathrm{~m}$ Telescopio Nazionale Galileo (TNG) with the Near-Infrared Camera Spectrometer (NICS; see Baffa et al. 2001). All three nights were photometric and had stable seeing conditions. Among the observing modes offered by the NICS and SpeX instruments are high-throughput, low-resolution spectroscopic modes with a prism disperser (Oliva 2000; Rayner et al. 2003). These modes yield a complete $0.8-2.4 \mu \mathrm{m}$ spectrum in a single exposure. With NICS a 1"5 width slit was used, corresponding to a spectral resolving power $\mathcal{R} \sim 34$, quasi-constant along the spectrum. With SpeX a 0".8 width slit was used, corresponding to a spectral resolving power $\mathcal{R} \sim 130$.

The object was relatively bright $\left(V_{\mathrm{mag}} \sim 10\right)$ and was identified as a moving object at the predicted position and with the expected motion. The guider images did not show any evidence of coma activity at the time. Because of the limited time available for these target-of-opportunity observations, we were unable to obtain imaging frames for quantitative limits on possible coma contribution to the flux. The slit was oriented in the parallactic angle (the position angle for which the slit is perpendicular to the horizon) to avoid problems with differential atmospheric refraction, and the tracking was at the comet's motion. At the IRTF, the data acquisition consisted of the following sequence: a $30 \mathrm{~s}$ exposure time image was obtained in one position (position $A$ ) of the slit, and then the telescope was offset by $10^{\prime \prime}$ in the direction of the slit (position $B$ ), where another $30 \mathrm{~s}$ exposure time image was obtained. This process was repeated, and $12 A B B A$ cycles were acquired. At the IRTF the total exposure time was $1440 \mathrm{~s}$ on each of the two nights. At the TNG, the acquisition process was the same, with three $A B B A$ cycles of $60 \mathrm{~s}$ exposures in each position for a total exposure time of $720 \mathrm{~s}$.

The reduction and calibration of all the near-IR spectra was done as described in Licandro et al. (2006). Flat-field images and wavelength calibrations were obtained periodically throughout the observations. To correct for telluric absorption and to

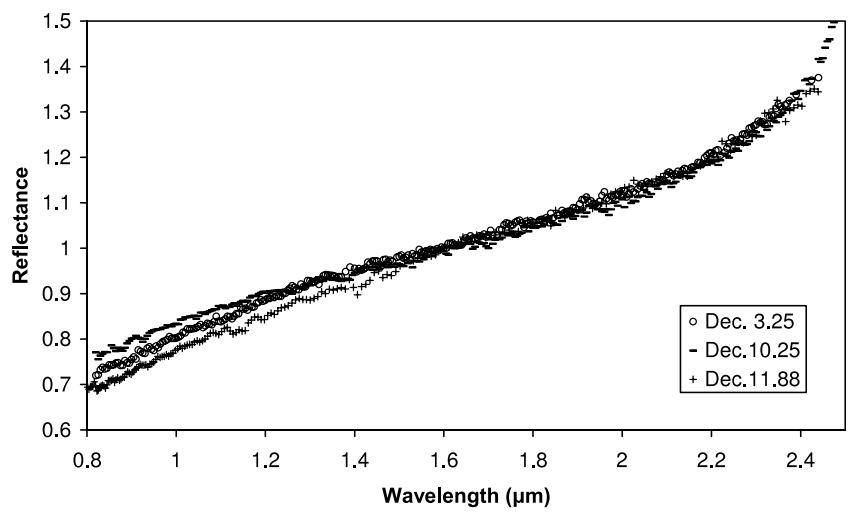

FIG. 1.-Near-IR reflectance spectra of comet 162P obtained on UT 2004 December 3.25 (circles), 10.25 (dashes), and 11.88 (plus signs) normalized to 1.0 at $1.60 \mu \mathrm{m}$. The difference between these spectra, although small, appears to be real and not due to systematic effects. obtain the relative reflectance, the G stars Landolt SA 115-271, Landolt SA 93-101, and Landolt SA 112-1333 (Landolt 1992), which have colors very similar to that of the Sun, were observed during the same nights and at a similar air mass as the comet. These stars were observed in previous nights and compared with the spectrum of the solar analog P330E (Colina \& Bohlin 1997), also observed by us. The Landolt stars and the solar analog showed similar spectra in the near-IR region; therefore, we used the Landolt stars as local solar analogs. The flat-fielded and wavelength-calibrated spectral frames were shifted to align and stacked to provide combined frames from which the spectra were extracted using the procedure described by Cushing et al. (2004). The near-IR spectra of the comet were divided by the mean spectrum of the solar analog stars and normalized to unity at $1.6 \mu \mathrm{m}$, thus obtaining the relative reflectances for all three dates plotted in Figure 1. Because the object was bright and the atmospheric conditions very good, the random errors for these spectra are particularly low (about $1 \%$ for each point); the pointto-point variability in each spectrum confirms this. For clarity, we do not show the error bars in Figure 1; instead, we plot one of our spectra (December 3) with the error bars for each point in Figure 2. The observational uncertainties for the other two nights are essentially identical; all three nights had equally good weather and the procedure was the same. To illustrate the potential impact of telluric absorptions, we plot the flux distribution (in counts vs. wavelength) for one of our standards in Figure 3. In principle, telluric conditions can vary between the comet spectra and the standard-star spectra, introducing false spectral features, particularly in the two deepest bands near 1.35-1.45 and $1.80-2.0 \mu \mathrm{m}$. However, this was not the case in our spectra, and false structure is no more than $2 \%$ above or below the continuum. Beyond $2.0 \mu \mathrm{m}$, the shape of all three near-IR spectra is dominated by the thermal contribution from the warm nucleus (which is used in Paper II to estimate the albedo and beaming parameter of the nucleus).

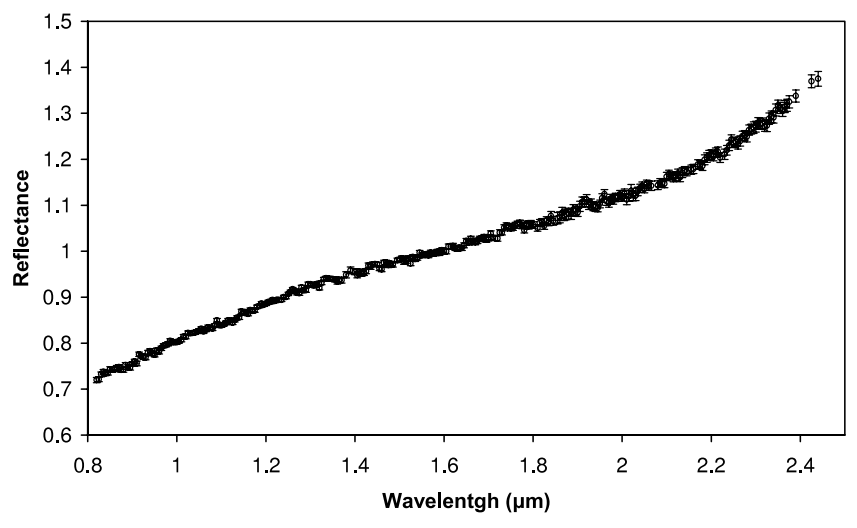

FIG. 2.-December 3.25 near-IR reflectance (from Fig. 1) with $1 \sigma$ error bars $(0.5 \%)$. 


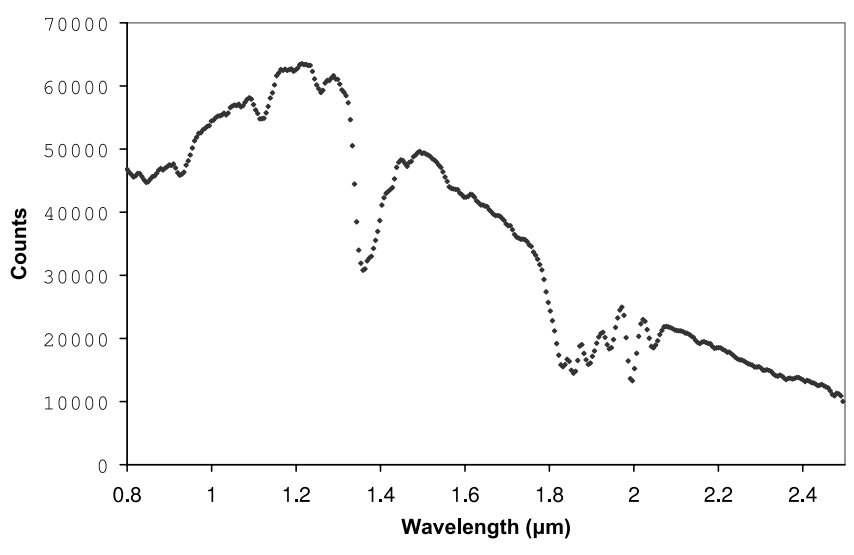

FIG. 3.-Flux (in counts) from one of the standards, Landolt SA 93-101, as a function of wavelength, illustrating the behavior of the atmospheric extinction.

The three near-IR spectra in Figure 1 are very similar; however, slight differences are apparent. The December 10 spectrum is consistently higher than those of December 3 and 11 shortward of $1.4 \mu \mathrm{m}$ and consistently lower longward of $1.6 \mu \mathrm{m}$. Similarly, the December 11 spectrum is consistently lower than those of December 3 and 10, shortward of $1.5 \mu \mathrm{m}$. These differences do not appear to be systematic artifacts. Although slight differences $(\sim 2 \%)$ in the slope can be attributed to systematic effects resulting from the centering of the object on the slit and the seeing conditions (Cushing et al. 2004), such slope differences would not mimic the observed differences. We consider these to be real changes in the spectrum of the comet produced by rotational variations of the surface composition and/or a variable coma contribution; however, we do not have the means to evaluate either of these possibilities. In any case, since the differences are relatively minor, we averaged the three near-IR spectra to combine them with the visible spectrum and for comparison with other objects and models $(\S 3)$. The slope of the averaged IR spectrum from 1 to $2 \mu \mathrm{m}$ and normalized at $1.6 \mu \mathrm{m}$ is $S^{\prime}=3.6 \% \pm$ $0.3 \%$ per $1000 \AA$ (Table 2). Note that the continuum slope of a spectrum can be parameterized using the normalized reflectivity gradient, which is usually denoted with $S^{\prime}\left[\%(1000 \AA)^{-1}\right]$ and defined as $S^{\prime}=d S / d \lambda / S^{\prime \prime}$ (e.g., Jewitt 2002). Here $S$ is the reflectivity (object flux density divided by the flux density of the Sun at the same wavelength) and $S^{\prime \prime}$ is the mean value of the reflectivity in the wavelength range over which $d S / d \lambda$ is computed. The gradient $S^{\prime}$ is used to express the percentage change in the strength of the continuum per $1000 \AA$. The use of $S^{\prime}$ facilitates comparisons with the spectral slopes of other objects at visible and IR wavelengths; however, one must keep in mind that $S^{\prime}$ only conveys the average slope of a spectrum that is not a straight line. Our estimates of the uncertainty in the value of $S^{\prime}$

TABLE 2

Spectral Slopes for 162P and Other Comet Nuclei

\begin{tabular}{|c|c|c|c|}
\hline Comet & $\begin{array}{c}\text { Spectral Slope } S^{\prime} \\
\left(\%[1000 \AA]^{-1}\right)\end{array}$ & $\begin{array}{c}\text { Normalization } \\
(\mu \mathrm{m})\end{array}$ & Notes \\
\hline 162P (vis)...................... & $9.2 \pm 1.0$ & 0.55 & \\
\hline $162 P$ (IR) .................... & $3.6 \pm 0.3$ & 1.6 & \\
\hline 19P/Borrelly.................. & $7.7 \pm 0.8$ & 1.6 & a \\
\hline 124P/Mrkos .................. & $3 \pm 1.0$ & 1.6 & $\mathrm{~b}$ \\
\hline $\mathrm{C} / 2001 \mathrm{OG}_{108} \ldots \ldots \ldots \ldots$ & $2.1 \pm 0.2$ & 1.6 & $\mathrm{a}$ \\
\hline
\end{tabular}

${ }^{\text {a }}$ Estimated from the plots in Soderblom et al. 2004 (19P/Borrelly) and Abell et al. $2005\left(\mathrm{C} / 2001 \mathrm{OG}_{108}\right)$.

${ }^{b}$ From Licandro et al. 2003.

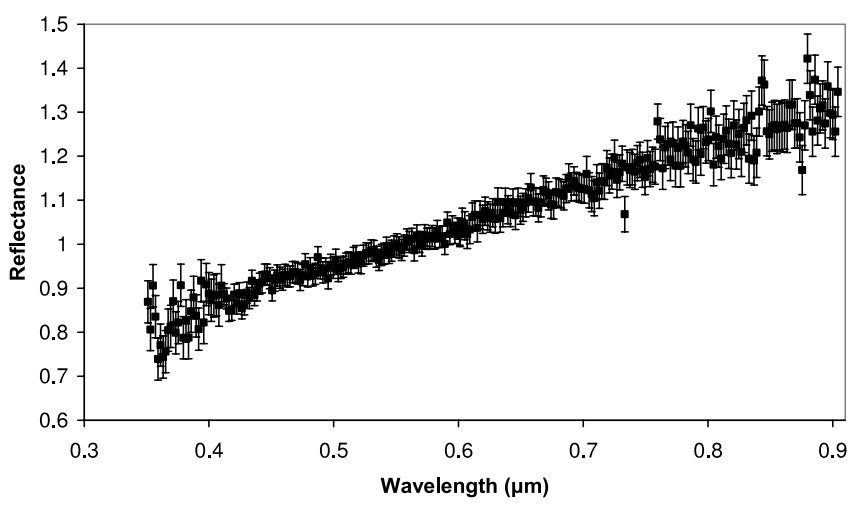

FIG. 4.-Visible reflectance spectrum obtained on December 12.86 with $1 \sigma$ error bars plotted normalized to 1.0 at $0.55 \mu \mathrm{m}$. An absorption at $0.42 \mu \mathrm{m}$ that is $0.02 \mu \mathrm{m}$ wide (FWHM) is marginally detected. This feature could be produced by hydrated silicates $(\S 3.1)$.

include contributions from small variations in the slopes of our standard stars. In Table 2 the value of $S^{\prime}$ for $124 \mathrm{P} /$ Mrkos is from Licandro et al. (2003). For comets 19P/Borrelly and C/2001 $\mathrm{OG}_{108}, S^{\prime}$ was estimated from plots in Soderblom et al. (2004) and Abell et al. (2005) $\left(\mathrm{OG}_{108}\right)$.

\subsection{Visible Spectrum}

A visible spectrum of our target was obtained on UT 2004 December 12.88 with the $2.5 \mathrm{~m}$ Nordic Optical Telescope (NOT) on La Palma, Spain, using the Andalucia Faint Object Spectrograph and Camera (ALFOSC) instrument. We used a 1".3 slit and grism disperser 4 with a wavelength range of $0.32-0.91 \mu \mathrm{m}$ ( $\lambda_{\text {cent }}=0.58 \mu \mathrm{m}$ and spectral resolving power $R=710$ ). Spectra in two wavelength ranges were obtained and combined. A $540 \mathrm{~s}$ exposure spectrum without a filter was obtained covering the $0.35-0.65 \mu \mathrm{m}$ region, and another spectrum with a secondorder blocking filter (cutoff wavelength at $0.47 \mu \mathrm{m}$ ) was obtained covering the $0.5-0.9 \mu \mathrm{m}$ region with an exposure time of $670 \mathrm{~s}$. The overlap region between the two spectra from 0.50 to $0.65 \mu \mathrm{m}$ gave excellent agreement and was used to combine them. As in the near-IR observations, the slit was oriented in the parallactic angle and the tracking was at the object proper motion. The spectral data reduction was done using the Image Reduction and Analysis Facility (IRAF) package, following standard procedures. Wavelength calibration was performed using a heliumneon lamp, and two solar analog stars (Landolt SA 98-978 and SAO 93936, also known as Hyades 64) were observed to correct for telluric absorption and to obtain the reflectance spectrum of the comet, as it was done in the near-IR. The reduction of this spectrum was done as described in de León et al. (2004). The calibrated visible spectrum of the comet was divided by each solar analog; both of these ratios were averaged for the final result shown in Figure 4. At visible wavelengths it is customary to normalize spectra at $0.55 \mu \mathrm{m}$. The slope of the visible spectrum of comet $162 \mathrm{P}$ normalized at $0.55 \mu \mathrm{m}$ is $S^{\prime}=9.2 \% \pm$ $1.0 \%$ per $1000 \AA$ ( Table 2 ). This value is roughly in the middle of the range of those observed for comet nuclei (e.g., Campins \& Fernández 2002). The overlap between 0.8 and $0.9 \mu \mathrm{m}$ for the visible and the near-IR spectra was used to combine these into the same reflectance scale normalized at $1.60 \mu \mathrm{m}$, thus obtaining the relative reflectance plotted in Figure 5.

\section{ANALYSIS AND RESULTS}

There are no strong spectral features in the wavelength range covered. This paucity of deep absorption features is common 


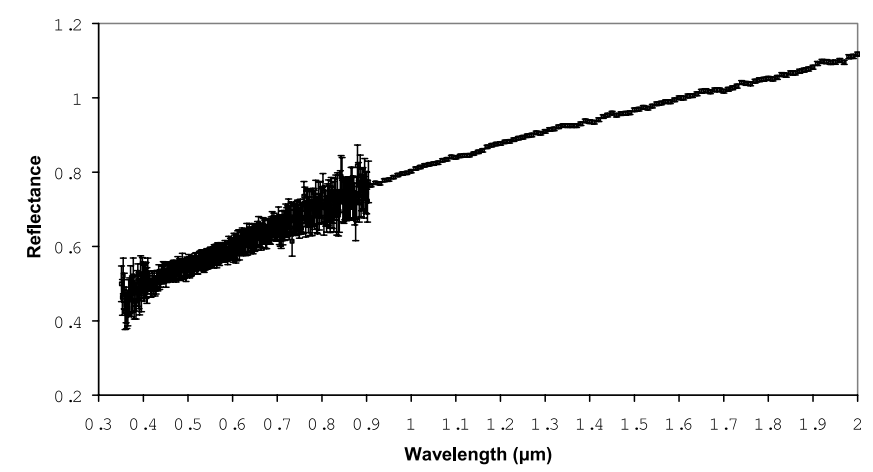

FIG. 5.-Visible reflectance spectrum (from Fig. 4) plotted with the average of all three near-IR reflectance spectra, normalized to 1.0 at $1.60 \mu \mathrm{m}$. The overlap between 0.8 and $0.9 \mu \mathrm{m}$ in the visible and near-IR spectra was used to combine both into the same reflectance scale. The reflectance beyond $2.0 \mu \mathrm{m}$ is dominated by thermal emission and not plotted here.

among low-albedo asteroids and the few comet nuclei observed in this spectral region (e.g., Abell et al. 2005; Campins et al. 2003; Emery \& Brown 2003; Licandro et al. 2002, 2003; Fernández et al. 2004; Soderblom et al. 2004). However, the overall shape of the spectrum can yield significant constraints on the surface composition. In addition, there is a hint of spectral structure that deserves some attention.

\subsection{Spectral Shape}

In the $0.3-0.9 \mu \mathrm{m}$ region the slope and shape of the spectrum are within the range found for D-type asteroids (e.g., Jewitt 2002; see also $\S 3.4$ ). Closer scrutiny of the visible spectrum (Fig. 4) shows hints of an absorption at 0.42 and $0.02 \mu \mathrm{m}$ wide (FWHM). This possible feature corresponds well with an absorption observed in a number of low-albedo asteroids, more specifically $\mathrm{C}$-, P-, and G-type asteroids, and attributed to hydrated minerals such as jarosite $\left[\mathrm{KFe}_{3}(\mathrm{SO} 4)_{2}(\mathrm{OH})_{6}\right]$ (Vilas et al. 1993; Cochran \& Vilas 1997). Although tantalizing, the feature is marginally detected, and it is difficult to come to any conclusion.

In the near-IR, we see no evidence for spectral structure greater than $2 \%$ of the continuum value. Jarosite, the mineral considered in the previous paragraph, has an absorption near $2.25 \mu \mathrm{m}$, which is absent in our spectra (Fig. 1). However, as mentioned in $\S 2$, that portion of our spectra has a strong thermal emission contribution, making the absence of the $2.25 \mu \mathrm{m}$ less significant.

\subsection{Comparison with Spectra of Other Comets}

Our averaged near-IR spectrum of $162 \mathrm{P}$ is plotted from 1 to $2 \mu \mathrm{m}$ in Figure 6, along with the near-IR spectra of comets 19P/ Borrelly, 124P/Mrkos, and LONEOS OG 108 (Soderblom et al. 2004; Licandro et al. 2003; Abell et al. 2005). Each of these comet spectra is featureless within the noise, and they have slopes $S^{\prime}=7.7 \%, 3.0 \%$, and $2.1 \%$ per $1000 \AA$, respectively (Table 2 ). The spectrum of $19 \mathrm{P} /$ Borrelly is very red, among the reddest observed in the solar system (the term "red" is commonly used to refer to spectra with increasing reflectance as a function of wavelength). We note that this spectrum was obtained by the Deep Space 1 mission, and its reduction and calibration presented unique challenges (Soderblom et al. 2004). There is an additional near-IR spectrum of a comet nucleus, that of $28 \mathrm{P} /$ Neujmin 1 (Licandro et al. 2001; Campins et al. 2001). The published nearIR spectrum of 28P/Neujmin 1 also appears to be featureless within the noise and has a nearly flat slope; however, it has been reported that 28P displays significant changes in its spectral slope (Campins et al. 2003). Therefore, a proper comparison with 28P

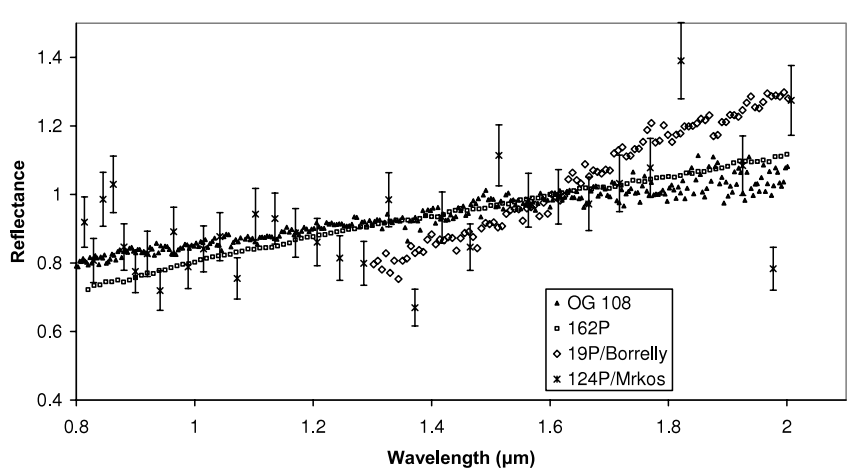

FIG. 6.-Near-IR reflectance spectrum of comet 162P (squares) plotted with the spectra of comets 19P/Borrelly (diamonds), 124P/Mrkos (crosses), and C/2001 $\mathrm{OG}_{108}$ (triangles) (Soderblom et al. 2004; Licandro et al. 2003; Abell et al. 2005), normalized to 1.0 at $1.60 \mu \mathrm{m}$. The spectrum of $124 \mathrm{P}$ was binned to a lower spectral resolution for clarity. Also for clarity, the error bars are only plotted for 124P; the point-to-point variations in the other spectra are more evident and are indicative of the uncertainty. The spectral slopes for these objects are listed in Table 2.

requires a better understanding of its variability. Asteroid 944 Hidalgo is in a cometary orbit and is considered among the most likely asteroids to be of cometary origin. Asteroid 944 Hidalgo also shows spectral variability in the near-IR, and a detailed discussion of Hidalgo's rotational variability is given in a separate paper (H. Campins et al. 2006, in preparation). Some of the near-IR spectra of Hidalgo are essentially identical to that of 162P/Siding Spring (Campins et al. 2005; Abell et al. 2005). From Figure 6 we conclude that comet nuclei show a range of red slopes in this wavelength region analogous to the variations observed at shorter wavelengths (Campins \& Fernández 2002 and references therein).

\subsection{Comparison with Spectra of Asteroids}

Low-albedo "primitive" asteroids in the outer main belt, as well as in the Trojan clouds, also have red spectra with little or no structure. By primitive asteroids we mean $\mathrm{C}, \mathrm{P}$, and $\mathrm{D}$ types in the Tholen classification scheme (Tholen \& Barucci 1989); some of these primitive asteroids may be related to comets. In Figure 7 we compare our visible and near-IR spectra of comet $162 \mathrm{P}$ with the average spectra of three representative main-belt P-type asteroids

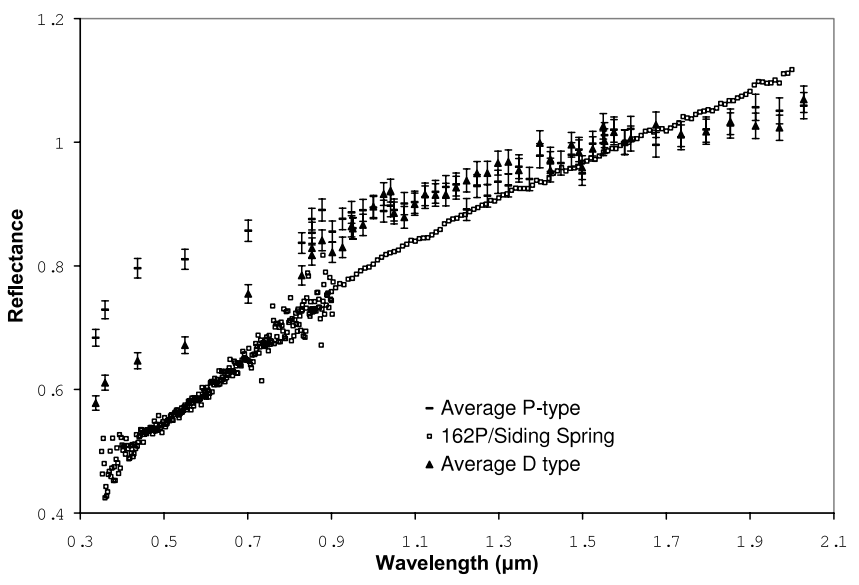

FIG. 7.- Visible and near-IR spectra of comet $162 \mathrm{P}$ plotted with the average spectra of three representative P-type asteroids (horizontal bars) and three representative D-type asteroids (triangles), normalized to 1.0 at $1.60 \mu \mathrm{m}$. In this case the error bars show the range of the asteroid spectra that were used in this average. In the visible region the slope of the comet and that of the $\mathrm{D}$ asteroids are the same, but in the near-IR the comet's slope is significantly steeper than that of the asteroids. 


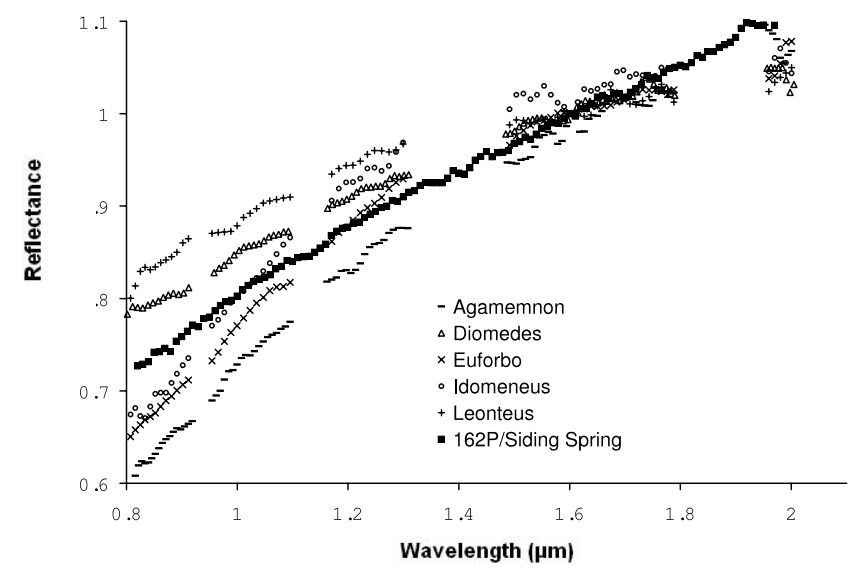

FIG. 8.-Near-IR spectrum of comet 162P (squares) plotted with the spectra of five Trojan asteroids from Emery \& Brown (2003), normalized to 1.0 at $1.60 \mu \mathrm{m}$. The spectrum of comet $162 \mathrm{P}$ is clearly within the range of spectral slopes of Trojan asteroids. For clarity, in this and further figures the comet error bars are not plotted.

(65 Cybele, 76 Freia, and 476 Hedwig) and three representative main-belt D-type asteroids (336 Lacadiera, 368 Haidea, and 773 Irmintraud; Zellner et al. 1985; Bell et al. 1988). Shortward of $1.0 \mu \mathrm{m}$ the spectrum of $162 \mathrm{P}$ is closer to that of D-type asteroids $\left(S^{\prime}=9.1 \% \pm 1.1 \%\right.$ per $1000 \AA$; Fitzsimmons et al. 1994). In the $1-2 \mu \mathrm{m}$ region, $162 \mathrm{P}$ is significantly redder than the $\mathrm{P}$ and $\mathrm{D}$ types plotted, which have $S^{\prime}$ values of $1.9 \%$ and $2.4 \% \pm 0.2 \%$ per $1000 \AA$, respectively.

Recently, Morbidelli et al. (2005) provided dynamical arguments indicating that Trojan asteroids may have formed in more distant regions and were subsequently captured into co-orbital motion with Jupiter. In addition, escaped Trojans are considered one possible source of Jupiter-family comets (e.g., Marzari et al. 1997). Since it is well established that the visible spectra of Trojans are similar to those of inactive comet nuclei (e.g., Jewitt 2002 and references therein), we concentrate on comparisons of near-IR spectra. In Figures 8 and 9 we compare our near-IR spectrum with those of seven of the Trojan asteroids observed at similar wavelengths by Emery \& Brown (2003). Figure 8 shows the range of spectra exhibited by the Trojan asteroids. In Figure 9 we present the near-IR spectra of two Trojan asteroids (1143 Odysseus and 2797 Teucer), which although not identical are closest to that of comet 162P. The $V$-band geometric albedos $\left(p_{v}\right)$ for these two Trojans are $7.5 \% \pm 0.5 \%$ and $6.2 \% \pm 0.5 \%$,

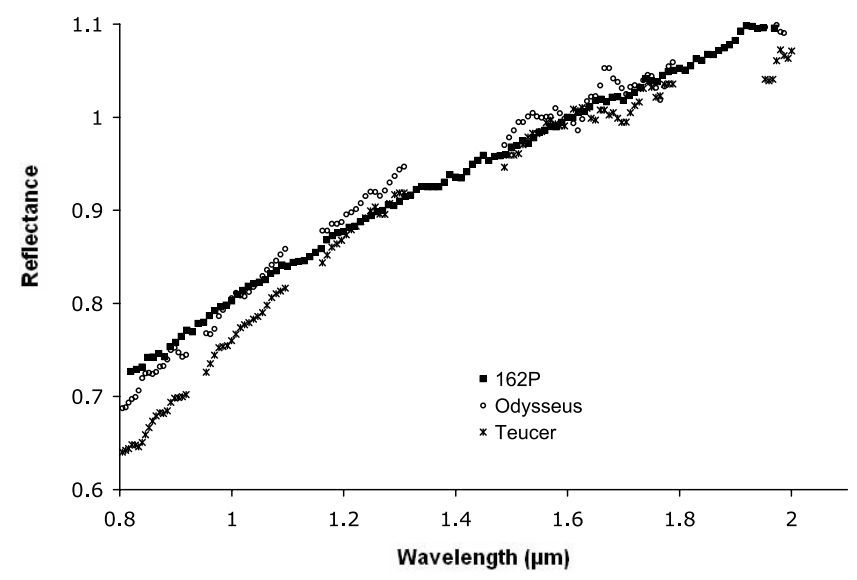

FIG. 9.- Near-IR spectrum of comet 162P (squares) plotted with the two Trojan asteroids from Emery \& Brown (2003) with the most similar spectral shapes, 1143 Odysseus and 2797 Teucer. The spectra are normalized to 1.0 at $1.60 \mu \mathrm{m}$.

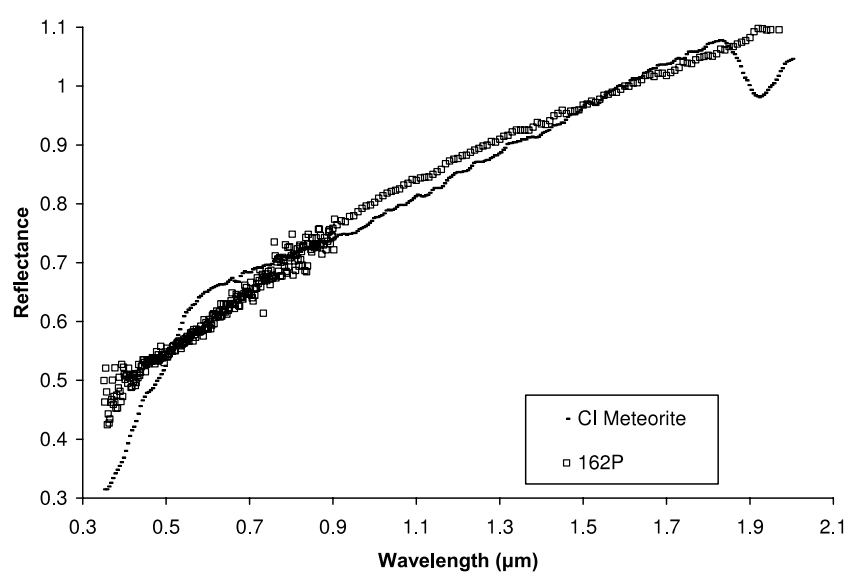

FIG. 10.- Visible and near-IR spectrum of comet 162P compared with that of a $\mathrm{C}$ I carbonaceous chondrite meteorite.

respectively, about twice the value for $162 \mathrm{P}\left(p_{v}=3.3 \% \pm 1.3 \%\right.$; Paper II). The spectral similarity and comparable albedos may be indicative of analogous compositions (see $\S 3.6$ ). At the same time, the spectral diversity among Trojan asteroids is also comparable with that seen among the few comet nuclei observed so far in the near-IR (Fig. 6). In other words, among Trojan asteroids we find reasonable matches to individual comet spectra, as well as to the spectral range observed so far among comet nuclei. Such similarities suggest that the formation environments of both populations may have indeed been analogous, as proposed by Morbidelli et al. (2005).

\subsection{Comparison with Centaurs and Trans-Neptunian Objects}

Comets are believed to be dynamically related to Centaurs and trans-Neptunian objects (TNOs), and near-IR spectra of these objects are increasingly available (see review by Barucci et al. 2005 and references therein). Some of these objects have featureless spectra in the near-IR, but others do not. The presence of strong absorption bands and the range of observed spectral characteristics suggest that many of the Centaurs and TNOs have surface compositions different from those found among comet nuclei (e.g., Cruikshank et al. 1998; Jewitt 2002; Licandro et al. 2001, 2002; Barucci et al. 2005). Possible processes that would explain such compositional differences are discussed in the references cited above. A detailed comparison of available near-IR spectra of Centaurs, TNOs, and comet nuclei is beyond the scope of this work.

\subsection{Comparison with Meteorite Spectra}

Comparison of the visible and near-IR spectra with meteoritic samples can help constrain the composition of comets. As is the case with comparisons between asteroids and meteorites, care must be taken, since terrestrial weathering can contaminate the meteoritic samples and space weathering can influence the spectra of asteroids (e.g., Clark et al. 2002). We used the Relab database (Pieters \& Hiroi 2004) to search for appropriate fits to our spectrum. The best fit to a primitive meteorite was obtained with the Alais C I carbonaceous chondrite (Relab sample MR-MJG106) and is shown in Figure 10. This fit is not very good, with the most significant shape discrepancies in the ultraviolet, visible, and $1.9 \mu \mathrm{m}$ regions. Surprisingly, the best fit was to an iron meteorite, as shown in Figure 11 (Relab sample SC-EAC-063). We consider this fit a coincidence rather than indicative of a compositional similarity for several reasons, including the fact that iron meteorites are the result of igneous processes incompatible with 


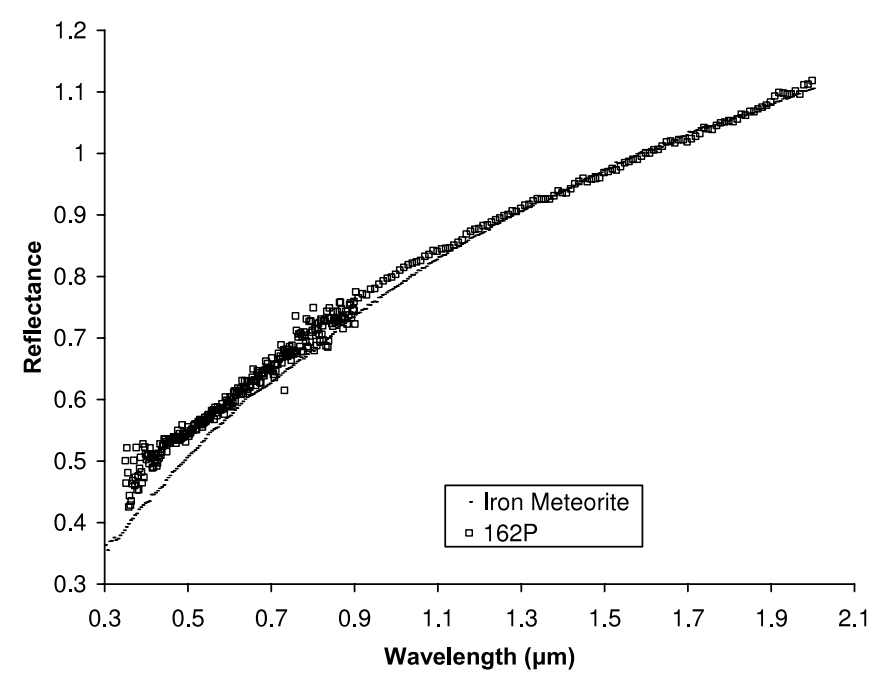

FIG. 11.-Visible and near-IR spectrum of comet 162P compared with an iron meteorite. This spectral fit is not indicative of compositional similarities $(\S 3.5)$.

the high abundance of volatiles in comets. In addition, the geometric albedo of this iron meteorite is $p_{v}=10.6 \%{ }^{10}$ (Pieters \& Hiroi 2004), 3 times greater than that of this $\operatorname{comet}\left(p_{v}=3.3 \% \pm\right.$ $1.3 \%$; Paper II). This spectral match to an unlikely material underscores the importance of using albedo in addition to spectra when constraining the surface composition of these objects. Campins \& Swindle (1998) conclude that comets can yield macroscopic meteorites, which have not either been found or recognized. As more comet and meteorite spectra become available, they will likely play an important role in the identification of cometary meteorites.

\subsection{Models}

Modeling of the surface spectra of atmosphereless solar system objects to infer their composition can be a powerful analytical tool and has been discussed in a number of publications (e.g., Hapke 1981; Shkuratov et al. 1999; Cruikshank et al. 1998; Emery \& Brown 2004; Clark et al. 2004). We modeled our visible and nearIR spectra using the scattering theory described by Shkuratov et al. (1999) for areal mixtures. Our approach is very similar to that used for modeling Trojan asteroids (Emery \& Brown 2004) and Centaurs (e.g., Cruikshank et al. 1998; Poulet et al. 2002). The main constraints are the albedo ( $p_{v}=3.3 \% \pm 1.3 \%$; Paper II) and the overall shape of the spectrum. The absence of strong

${ }^{10}$ The uncertainty in the albedo of the meteorite is negligible because it is calibrated in the laboratory with respect to a registered standard. spectral features prevents the identification of specific minerals, and the resulting model compositions are not unique. Furthermore, to include a mineral or molecule in our model it is necessary to have its optical constants, which are available for a limited set of likely components. If taken literally, our models would suggest that the surface of this comet could be up to $95 \%$ carbon (Table 3). This is unlikely, since carbon-rich meteorites (with albedos as low as that of this comet) have at most $3.2 \%$ by weight of carbon (Hutchison 2004, p. 29). Therefore, these models are not meant to strictly define the surface composition but rather to be indicative of likely components and to allow comparisons with related objects that have been modeled in similar ways.

The components we included are cosmochemically likely materials used to fit spectra of Trojan asteroids. Since the spectrum of $162 \mathrm{P}$ is similar to that of several Trojan asteroids (Figs. 8 and 9), we did not have to explore the whole parameter space and concentrated on mixtures that yielded good approximations to the spectra of these asteroids (Emery \& Brown 2004 and references therein). We have achieved reasonable fits to our spectra of $162 \mathrm{P}$ using mixtures that include the following components: (1) Two types of amorphous carbon. Amorphous carbons can exhibit extreme variations in their optical constants; we chose two specific types because of their complementary spectral characteristics (Figs. $12 a$ and $12 b$ ). The optical constants and production conditions for these two amorphous carbons are described in Rouleau \& Martin (1991) and Preibisch et al. (1993), respectively. (2) Silicates, more specifically pyroxene $\left[(\mathrm{Fe}, \mathrm{Mg}) \mathrm{SiO}_{3}\right]$, as described in Dorschner et al. (1995). (3) Organics, mainly Triton tholins. Tholins are the refractory residue resulting from plasma discharges in simple molecular mixtures. Triton tholin is the result of such a discharge in a gaseous mixture $\left(99.9 \% \mathrm{~N}_{2}\right.$, $0.1 \% \mathrm{CH}_{4}$ ) approximating the atmosphere of Triton (McDonald et al. 1994).

In Figure 12 we present six models with the compositions and particle sizes given in Table 3. In order to illustrate the approach that yielded the best fits (which we optimized visually), we first plot the individual spectra of each of the two amorphous carbon components in Figures $12 a$ and $12 b$. As observed in Figure 12c, these two components already give a reasonably good fit, with the main departures occurring at the shorter wavelengths. The fit by the carbon mix is independent of particle size (in the $10-500 \mu \mathrm{m}$ range). The addition of either organics (Triton tholin) or silicates (pyroxene with equal abundance of $\mathrm{Fe}$ and $\mathrm{Mg}$, labeled as $\mathrm{P} 7$ in Emery \& Brown 2004) to the carbon mix produces improved fits, as seen in Figures $12 d$ and $12 e$. The final fit in Figure $12 f$ includes all four components: two carbons, Triton tholin, and pyroxene. The grain size assumed was $10 \mu \mathrm{m}$ for both carbons, and grain size only became an important factor when considering the

TABLE 3

Model Surface Compositions

\begin{tabular}{|c|c|c|c|c|c|c|c|}
\hline \multirow[b]{2}{*}{ Model } & \multirow[b]{2}{*}{$\begin{array}{c}\text { ALBEDO } p_{v} \\
(\%)\end{array}$} & \multirow[b]{2}{*}{$\begin{array}{c}\text { AMORPHOUS CARBON } 1 \\
(\%)\end{array}$} & \multirow[b]{2}{*}{$\begin{array}{c}\text { AMORPHOUS CARBON } 2 \\
(\%)\end{array}$} & \multicolumn{2}{|c|}{ Triton Tholin } & \multicolumn{2}{|c|}{ PyroXene } \\
\hline & & & & Percentage & $\begin{array}{l}\text { Size } \\
(\mu \mathrm{m})\end{array}$ & Percentage & $\begin{array}{l}\text { Size } \\
(\mu \mathrm{m})\end{array}$ \\
\hline A & 4.0 & 100 & $\ldots$ & $\ldots$ & $\ldots$ & $\ldots$ & $\ldots$ \\
\hline В & 4.4 & $\ldots$ & 100 & $\ldots$ & $\ldots$ & $\ldots$ & $\ldots$ \\
\hline $\mathrm{C}$ & 4.3 & 62 & 38 & $\ldots$ & $\ldots$ & $\ldots$ & $\ldots$ \\
\hline 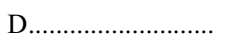 & 3.9 & 45 & 40 & $\ldots$ & $\ldots$ & 15 & 40 \\
\hline 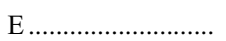 & 4.1 & 30 & 65 & 5 & 15 & $\ldots$ & $\ldots$ \\
\hline F & 3.9 & 44 & 38 & 3 & 50 & 15 & 200 \\
\hline
\end{tabular}




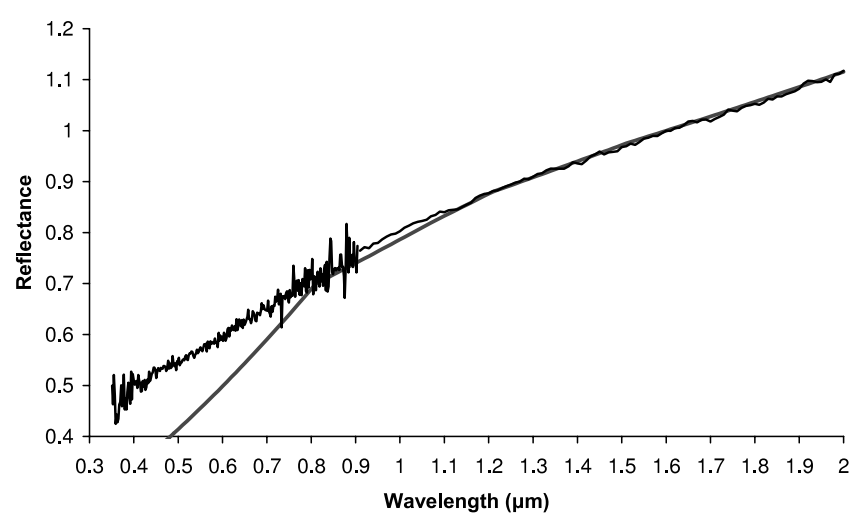

FIG. $12 a$

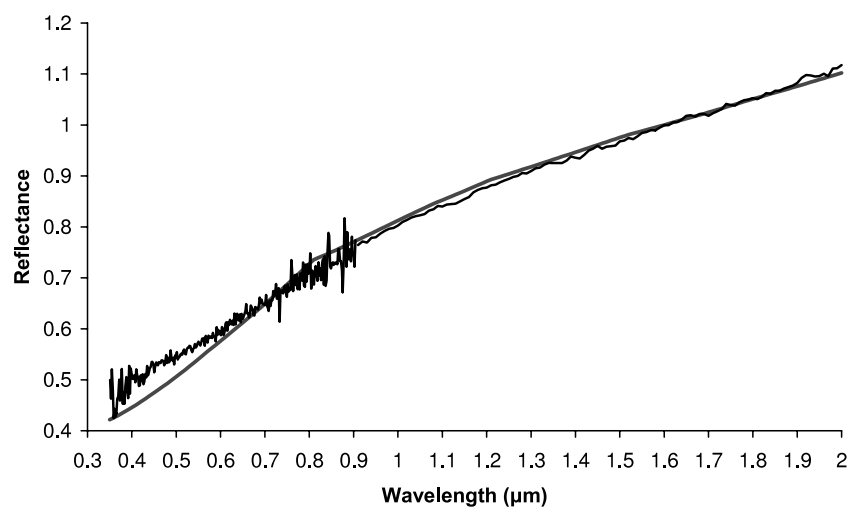

FIG. $12 c$

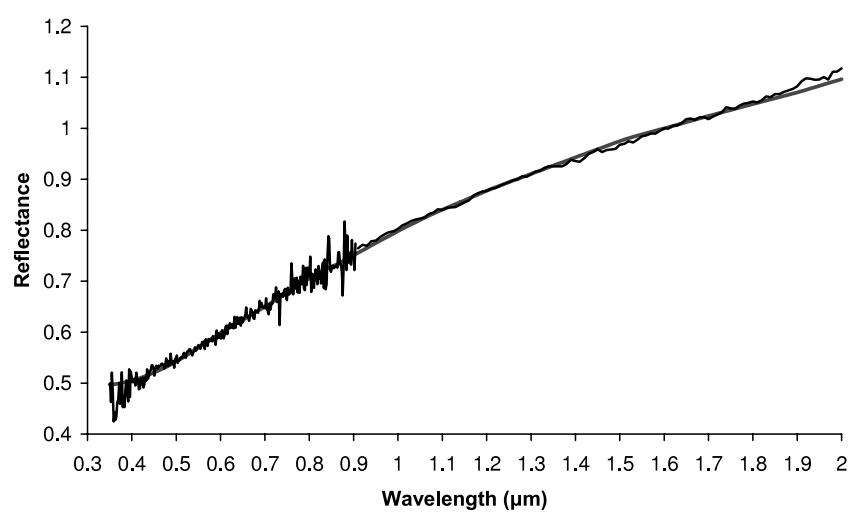

FIG. $12 e$

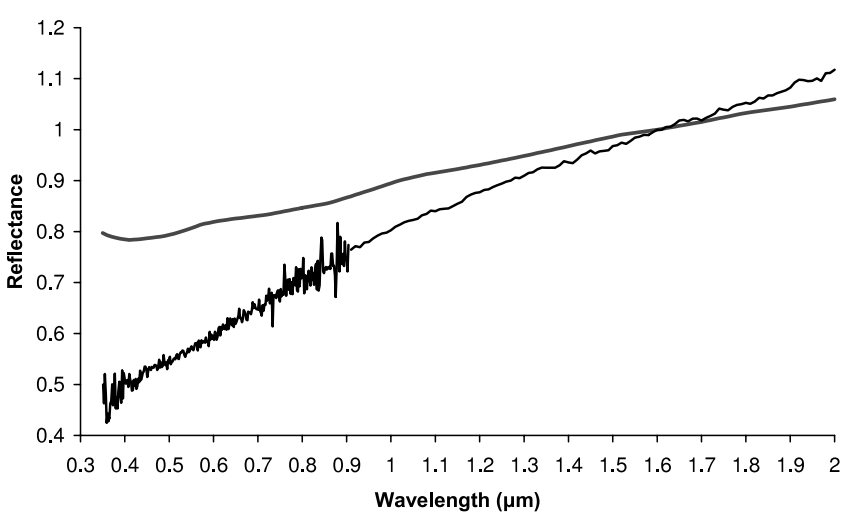

FIG. $12 b$

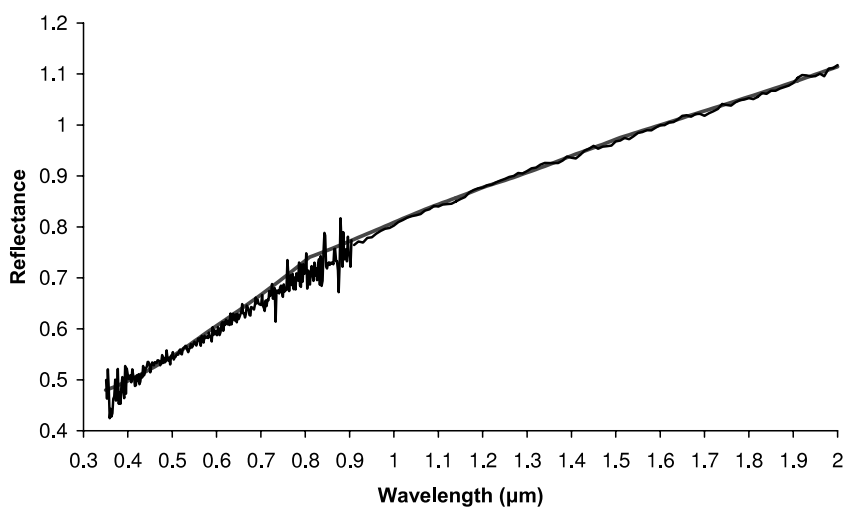

FIG. $12 d$

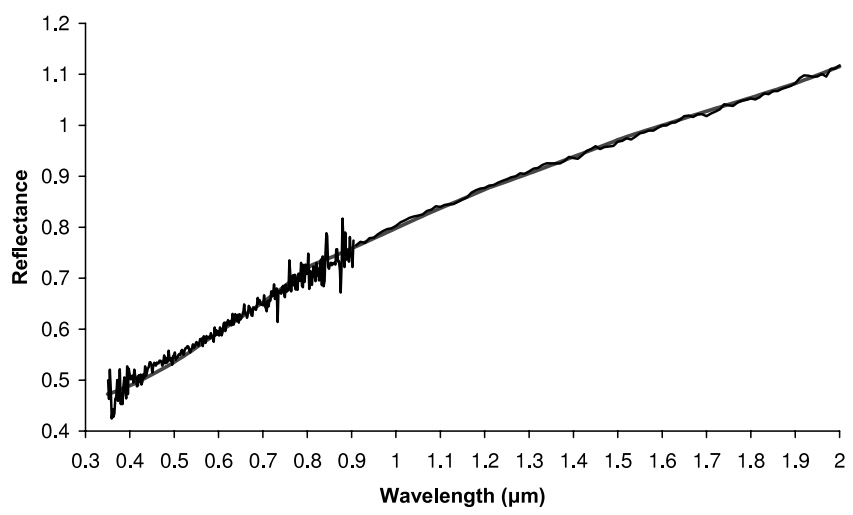

FIG. $12 f$

Fig. 12.-Models from Table 3, along with our spectrum of comet 162P, normalized to 1.0 at $1.60 \mu \mathrm{m}$. [See the electronic edition of the Journal for a color version of this figure.]

organics and silicates. All the models yield a geometric albedo within the uncertainties of that measured for the nucleus of this comet (Table 3). As seen in Figure 12, the amorphous carbons contribute to the overall shape and help lower the albedo. The pyroxene helped achieve the red slope at the longest wavelengths but is consistently too high from 0.7 to $1.0 \mu \mathrm{m}$. The Triton tholin (McDonald et al. 1994) fits well at most wavelengths except the shortest.

As mentioned, these initial models have significant limitations. The use of carbons with albedo close to that of our object required most of the surface to be carbon. More rigorous modeling should include carbon species with lower albedo and other components such as the hydrated silicate jarosite mentioned above (for which we did not have optical constants). Rotationally resolved visible and near-IR spectra would also be important to establish whether there are variations in surface composition as we found in 944 Hidalgo (H. Campins et al. 2006, in preparation).

It is important to point out that organics, including the Triton tholin used in our model, have strong absorptions in the 3-4 $\mu \mathrm{m}$ region due to $\mathrm{O}-\mathrm{H}, \mathrm{N}-\mathrm{H}$, and $\mathrm{C}-\mathrm{H}$ bonds (e.g., McDonald et al. 1994). These absorptions are not present in the spectra of the Trojan asteroids observed so far (e.g., Emery \& Brown 2003, 2004), suggesting that organics may not be primarily responsible for the red slope in the spectra of Trojan asteroids. However, 
organic solids have indeed been detected in the comae of several comets, including most recently in comet $\mathrm{P} /$ Tempel 1, particularly after the encounter and impact with the Deep Impact spacecraft (A'Hearn et al. 2005). Observations of comet 162P in the 3-4 $\mu \mathrm{m}$ region during this apparition might not have been diagnostic of the presence of organics. As mentioned in $\S 2.1$, beyond $2 \mu \mathrm{m}$ the shape of the spectrum of $162 \mathrm{P}$ is dominated by a strong thermal emission component. In future apparitions of comet 162P (orbital period $=5.3 \mathrm{yr}$ ) it would be useful to attempt observations in the 3-4 $\mu \mathrm{m}$ region when it is sufficiently far from the Sun for that spectral range not to be dominated by thermal emission. Such observations would be more diagnostic not only of organic components but also of hydrated silicates.

\section{CONCLUSION}

Our observations of the nucleus of comet $162 \mathrm{P}$ provide additional evidence that bare comet nuclei have spectra with muted or absent structure and low albedos. So far, the nuclei of comets appear spectrally similar to primitive asteroids, primarily D-type asteroids. The few comet nuclei observed spectroscopically in the near-IR exhibit considerable diversity, similar to that found among near-IR spectra of Trojan asteroids by Emery \& Brown (2003). We found reasonably good matches among Trojan asteroids to the spectral shape of comet 162P. Such similarities are consistent with an analogous formation environment for Trojan asteroids and Jupiter-family comets, as proposed by Morbidelli et al. (2005). No suitable spectral matches to our target were found among chondritic meteorites. We modeled the surface composition of our target using the spectral shape and albedo as constraints. Although the lack of sharp spectral structure makes the identification of specific surface components difficult, our models suggest amorphous carbon, pyroxenes, and/or organic compounds as likely components of the surface of comet 162P.

We thank J. Emery and P. Abell for providing their original data and for helpful comments. H. C. was supported by grants from NASA's Planetary Astronomy and from the National Science Foundation. J. Z. was supported by a fellowship from the Florida Space Grant Consortium and by a grant from the National Science Foundation. Y. R. F. acknowledges the support of a SIRTF Fellowship in performing some of this work. T. M. D. acknowledges the support of a postdoctoral fellowship from the Brazilian government's CNPq. Some of the data presented here have been taken using ALFOSC, which is owned by the Instituto de Astrofisica de Andalucia (IAA) and operated at the Nordic Optical Telescope under agreement between the IAA and the NBIfAFG of the Astronomical Observatory of Copenhagen.
Abell, P. A., et al. 2005, Icarus, 179, 174

A'Hearn, M. F., et al. 2005, Science, 310, 258

Baffa, C., et al. 2001, A\&A, 378, 722

Barucci, M. A., Doressoundiram, A., \& Cruikshank, D. P. 2005, in Comets II, ed. M. Festou, H. Keller, \& H. Weaver (Tucson: Univ Arizona Press), 647

Bell, J. F., Owensby, P. D., Hawke, B. R., \& Gaffey, M. J. 1988, in Lunar \& Planetary Science XIX, ed. G. Ryder \& V. L. Sharpton (Houston: Lunar Planet. Inst.), 57

Campins, H., \& Fernández, Y. 2002, Earth Moon Planets, 89, 117

Campins, H., Licandro, J., Chamberlain, M., \& Brown, R. H. 2001, BAAS, 33, 1094

Campins, H., Licandro, J., Guerra, J., Chamberlain, M., \& Pantin, E. 2003, AAS DPS Meeting, 35, 4702

Campins, H., Licandro, J., Ziffer, J., Fernández, Y., Hora, J., Kassis, M., \& Pinilla-Alonso, N. 2005, AAS DPS Meeting, 37, 1602

Campins, H., \& Swindle, Z., 1998, Meteoritics Planet. Sci., 33, 1201

Carusi, A., Kresak, L., Perozzi, E., \& Valsecchi, G. B. 1987, A\&A, 187, 899

Clark, B. E., Bus, S. J., Rivkin, A. S., Shepard, M. K., \& Shah, S. 2004, AJ, 128,3070

Clark, B. E., Hapke, B., Pieters, C., \& Britt, D. 2002, in Asteroids II, ed. R. Binzel et al. (Tucson: Univ. Arizona Press), 585

Cochran, A. L., \& Vilas, F. 1997, Icarus, 127, 121

Colina, L., \& Bohlin, R. 1997, AJ, 113, 1138

Cruikshank, D. P., et al. 1998, Icarus, 135, 389

Cushing, M. C., Vacca, W. D., \& Rayner, J. T. 2004, PASP, 116, 362

de León, J., Duffard, R., Licandro, J., \& Lazzaro, D. 2004, A\&A, 422, L59

Dorschner, J., Begemann, B., Henning, T., Jaeger, C., \& Mutschke, H. 1995, A\&A, 300, 503

Emery, J. P., \& Brown, R. H. 2003, Icarus, 164, 104 2004, Icarus, 170, 131

Fernández, Y. R., Campins, H., Kassis, M., Hora, J. L., Hergenrother, C. W., Binzel, R. P., \& Licandro, J. 2006, AJ, 132, 1354 (Paper II)

Fernández, Y. R., Lisse, C. M., Schleicher, D. G., Bus, S. J., Kassis, M., Hora, J. L., \& Deutsch, L. K. 2004, AAS DPS Meeting, 36, 2104

Fitzsimmons, A., Dahlgren, M., Lagerkvist, C.-I., Magnusson, P., \& Williams, I. P. 1994, A\&A, 282, 634

Hapke, B. 1981, J. Geophys. Res., 86, 4571

\section{REFERENCES}

Hsieh, H. H., Jewitt, D. C., \& Fernández, Y. R. 2004, AJ, 127, 2997

Hutchison, R. 2004, Meteorites (Cambridge: Cambridge Univ. Press)

Jewitt, D. 2002, AJ, 123, 1039

Landolt, A. U. 1992, AJ, 104, 340

Levison, H. F. 1996, in ASP Conf. Ser. 107, Completing the Inventory of the Solar System, ed. T. W. Rettig \& J. M. Hahn (San Francisco: ASP), 173

Licandro, J., Campins, H., Hergenrother, C., \& Lara, L. M. 2003, A\&A, 398, L45

Licandro, J., Guerra, J. C., Campins, H., DiMartino, M., Lara, L. M., Gil-Hutton, R., \& Tozzi, G. P. 2002, Earth Moon Planets, 90, 495

Licandro, J., Oliva, E., \& Di Martino, M. 2001, A\&A, 373, L29

Licandro, J., Pinilla-Alonso, N., Pedani, M., Oliva, E., Tozzi, G. P., \& Grundi, W. M. 2006, A\&A, 445, L35

Mallia, F., Masi, G., Wilcox, R., \& Lacruz, J. 2004, IAU Circ., 8436, 1

Marzari, F., Scholl, H., Tomasella, L., \& Vanzani, V. 1997, Planet. Space Sci., 45,337

Masi, G. 2004, IAU Circ., 8439, 1

McDonald, G. D., Thompson, W. R., Heinrich, M., Khare, B. N., \& Sagan, C. 1994, Icarus, 108, 137

Morbidelli, A., Levison, H. F., Tsiganis, K., \& Gomes, R. 2005, Nature, 435, 462

Oliva, E. 2000, Mem. Soc. Astron. Italiana, 71, 861

Pieters, C. M., \& Hiroi, T. 2004, in Lunar \& Planetary Science XXXV, ed. S. J. Mackwell (Houston: Lunar Planet. Inst.), 1720

Poulet, F., Cuzzi, J. N., Cruikshank, D. P., Roush, T., \& Dalle Ore, C. M. 2002, Icarus, 160,313

Preibisch, T., Ossenkopf, V., Yorke, H. W., \& Henning, T. 1993, A\&A, 279, 577

Rayner, J. T., Toomey, D. W., Onaka, P. M., Denault, A. J., Stahlberger, W. E., Vacca, W. D., Cushing, M. C., \& Wang, S. 2003, PASP, 115, 362

Rouleau, F., \& Martin, P. G. 1991, ApJ, 377, 526

Shkuratov, Y., Starukhina, L., Hoffman, H., \& Arnold, G. 1999, Icarus, 137, 235

Soderblom, L. A., Britt, D. T., Brown, R. H., Buratti, B. J., Kirk, R. L., Owen, T. C., \& Yelle, R. V. 2004, Icarus, 167, 100

Tholen, D. J., \& Barucci, M. A. 1989, in Asteroids II, ed. R. Binzel et al. (Tucson: Univ. Arizona Press), 208

Vilas, F., Larson, S. M., Hatch, E. C., \& Jarvis, K. S. 1993, Icarus, 105, 67

Weissman, P. R., Bottke, W. F., Jr., \& Levison, H. F. 2002, in Asteroids III, ed. W. Bottke et al. (Tucson: Univ. Arizona Press), 669

Zellner, B., Tholen, D. J., \& Tedesco, E. F. 1985, Icarus, 61, 355 